\title{
SOLAR POWER FOR NEAR-SUN, HIGH-TEMPERATURE MISSIONS
}

\author{
Geoffrey A. Landis \\ NASA John Glenn Research Center, \\ 21000 Brookpark Road, \\ Cleveland, $\mathrm{OH} 44135$
}

\begin{abstract}
Existing solar cells lose performance at the high temperatures encountered in Mercury orbit and inward toward the sun. For future missions designed to probe environments close to the sun, it is desirable to develop array technologies for high temperature and high light intensity. Approaches to solar array design for near-sun missions include modifying the terms governing temperature of the cell and the efficiency at elevated temperature, or use of techniques to reduce the incident solar energy to limit operating temperature. An additional problem is found in missions that involve a range of intensities, such as the Solar Probe + mission, which ranges from a starting distance of $1 \mathrm{AU}$ from the sun to a minimum distance of 9.5 solar radii, or $0.044 \mathrm{AU}$. During the mission, the solar intensity ranges from one to about 500 times AM0. This requires a power system to operate over nearly three orders of magnitude of incident intensity.
\end{abstract}

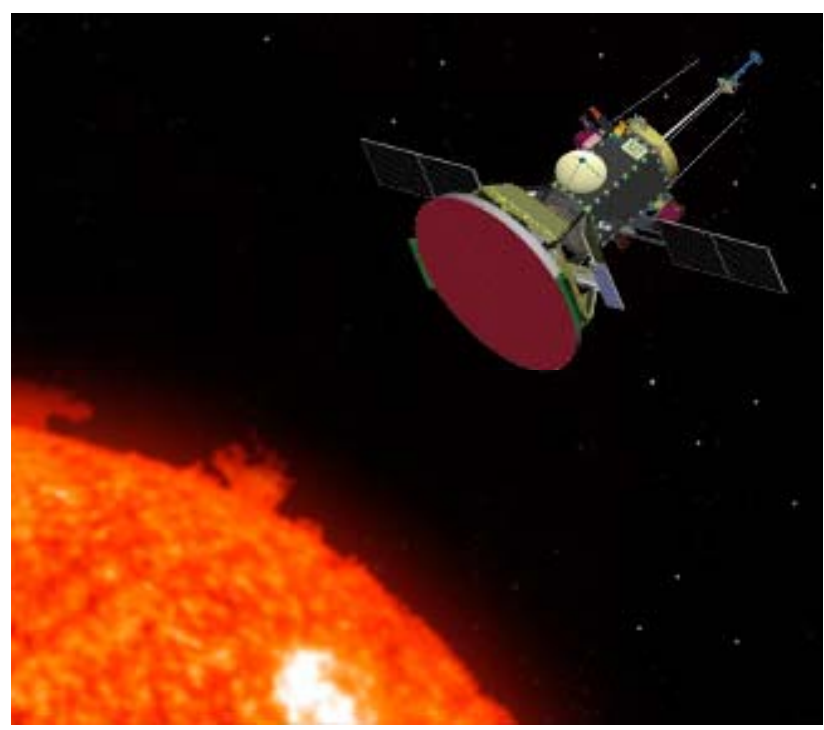

Figure 1: Solar Probe+, a mission to explore the sun's corona. This spacecraft is designed to be operated on photovoltaic power as close as $0.044 \mathrm{AU}$ from the sun. The flat solar shield protects the instrument package from the thermal environment near the sun. (Artist's conception, courtesy http://solarprobe.gsfc.nasa.gov).

\section{MISSIONS}

Extending the temperature range of operation for solar arrays is highly desirable for extending the range of operation of space missions to the near-sun environment [1-3]. Achieving high-efficiency and reliable operation in these temperature regimes is a difficult technological challenge. Existing solar cells suffer significant performance loss at the high temperatures encountered in Mercury orbit and near the sun.

An example of such a mission is the Solar Probe+ spacecraft [4], shown in Figure 1. This is a mission designed to probe the solar corona and the near-sun environment from an approach distance as close as 9.5 solar radii, or 0.044 Astronomical Units (AU). During the mission, the solar intensity is ranges from one to about 510 times AMO [5]. This requires a power system that can operate over nearly three orders of magnitude of incident intensity.

\section{SOLAR CELL OPERATING TEMPERATURE AND EFFICIENCY}

If future missions designed to probe environments close to the sun will be able to use photovoltaic power generation, solar cells that can function at high temperatures, under high light intensity, and high radiation conditions must be developed. The significant problem is that solar cells lose performance at high temperatures.

The operating temperature of a solar cell depends on fourth root of the incident intensity, as well as the ratio of solar absorptivity alpha $(\alpha)$ to thermal emissivity epsilon $(\varepsilon)$. According to radiative balance, if $\mathrm{I}$ is the incident intensity, the thermal radiation from the array must equal the absorbed solar radiation:

$$
\alpha \mathrm{I}=\left(\varepsilon_{\mathrm{f}}+\varepsilon_{\mathrm{r}}\right) \sigma \mathrm{T}^{4}
$$

where $T$ is the operating temperature in absolute (Kelvin) units, and the subscripts $f$ and $r$ indicate the emissivity from the front and rear sides of the cell, to account for the fact that the array can radiate waste heat away from both front and back sides $\left(\varepsilon_{r}\right.$ should be left out for a array with no back-side radiation). For convenience, here we define the absorptivity $\alpha$ as the net energy absorption, incorporating a factor of $(1-\eta)$ to account for the fact that the fraction of incident energy that is converted to electricity is not absorbed by the cell. Thus the equilibrium operating temperature $T$ is

$$
\mathrm{T}=\left[\left(\alpha /\left(\left(\varepsilon_{\mathrm{f}}+\varepsilon_{\mathrm{r}}\right) \sigma\right)\right) \mathrm{I}\right]^{1 / 4}=\mathrm{c} \mathrm{I}^{1 / 4}
$$

where the constant $\mathrm{c}$ is defined as

$$
c=\left[\alpha /\left(\left(\varepsilon_{\mathrm{f}}+\varepsilon_{\mathrm{r}}\right) \sigma\right)\right]^{1 / 4}
$$

Note that since front and back side of the cell need 
not have identical radiative properties in the infrared, the value of $\varepsilon$ is the average of front and back side properties.

The intensity depends on distance from the sun:

$$
\mathrm{I}=\mathrm{Io} / \mathrm{r}^{2}
$$

where Io is the solar intensity at Earth's distance from the sun, one $A U$, and distance $r$ is measured in $A U$. This equilibrium temperature is shown as a function of distance from the sun in Figure 2 for various values of the ratio of the $\alpha$ to $\varepsilon$ (where $\varepsilon$ here is the average of the front and back emissivity).

For the case of a planetary orbiter, the temperature is somewhat greater, since the solar array is heated not only by the incident solar flux, but also by solar flux reflected by the planet, as well as infrared emitted by the planet. This additional heating becomes more significant as the orbital altitude decreases. In the lowest orbit limit, this results in about twice the incident intensity, and hence an absolute temperature increase by a factor of the fourth root of 2 , or 1.19 .

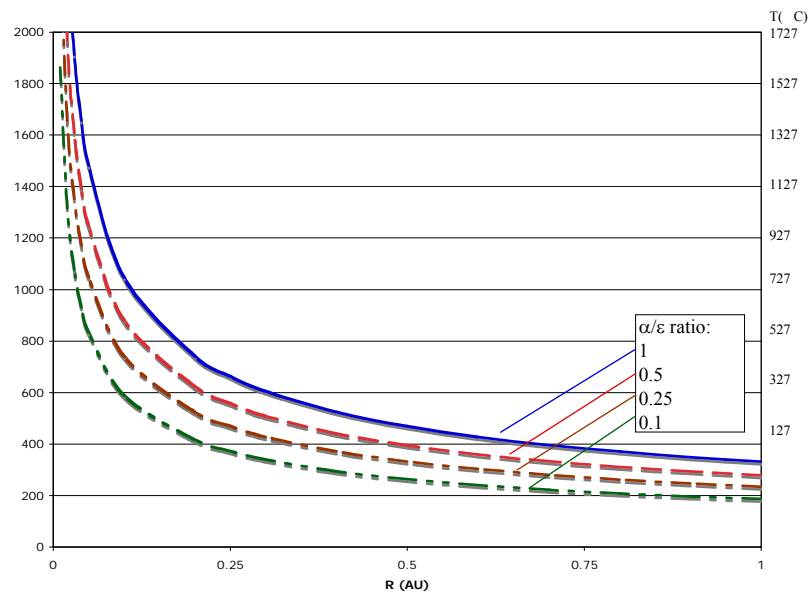

Figure 2: Temperature as a function of distance from the sun, for various values of $\alpha / \varepsilon$ ratio.

A solar cell's (unnormalized) temperature coefficient of efficiency $\kappa$ is defined as

$$
\kappa=d \eta / d T
$$

and the power at a temperature $T$ can be compared to the power output from a reference temperature, typically $27^{\circ} \mathrm{C}$ $(300 \mathrm{~K})$ by a linear extrapolation:

$$
P=\left(\eta_{300 \mathrm{~K}}+\kappa \Delta \mathrm{T}\right) \mathrm{I}
$$

In general, the temperature coefficient $\kappa$ is negative, corresponding to decrease in performance with increasing temperature. It is composed of separate parts corresponding to the change in short circuit current, open circuit voltage, and fill-factor of the cell. Of these, the coefficient of open-circuit voltage is typically the largest contribution [6]. The temperature coefficient is not a constant, but varies slightly with both intensity and temperature [7], however, these variations are typically small in the operational range of interest, and can be ignored in a first-level analysis.

From this, the power output from a cell is as a nonlinear function of the intensity. Defining $\eta_{\mathrm{o}}$ as the efficiency linearly extrapolated to $\mathrm{OK}$, which is calculated from the efficiency at $27 \mathrm{C}$ as

$$
\begin{aligned}
& \quad \eta_{\mathrm{o}}=\eta_{300 \mathrm{~K}}-300 \kappa \\
& \text { we get: } \quad \mathrm{P}=\mathrm{I}\left(\eta_{\mathrm{o}}+\kappa \mathrm{T}\right)=\mathrm{I}\left(\eta_{\mathrm{o}}+\kappa \mathrm{cI} \mathrm{I}^{1 / 4}\right)=\mathrm{I} \eta_{\mathrm{o}}+\kappa \mathrm{CI}^{5 / 4} .
\end{aligned}
$$

Figure 3 shows the calculated curve of power output as a function of temperature, comparing a high-efficiency silicon solar cell with a wide-bandgap solar cell. For any given solar cell technology, there exists an incident intensity above which the solar cell output decreases with increased intensity. In the linear model of equation 7 , this intensity is

$$
\text { I peak-output }=\left(-\eta_{\mathrm{o}} / \kappa \mathrm{c}\right)^{4}
$$

The temperature coefficient is, in general, nonlinear. This factor is accounted for in more detailed modeling. Since it depends on the difference between the open circuit voltage of the cell and the bandgap voltage [6], $\kappa$ decreases in magnitude proportionally to the Voc, which increases as the logarithm of the intensity. This moves the value of the peak output to slightly higher intensity.

For multijunction cell technologies, the voltage component of the temperature coefficient is the sum of the voltage dependence of the individual subcells. This will have a significant dependence on temperature as the lowest bandgap subcells in the series drop out as the temperature increases.

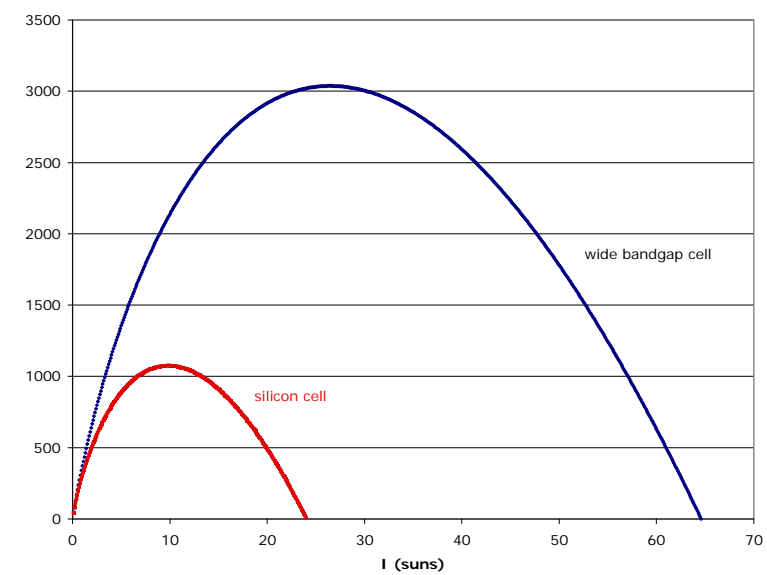

Figure 3: Curve of power output (normalized) as a function of intensity, assuming linear temperature coefficient.

The question of how to modify the temperature coefficient of solar cells has been addressed in earlier studies. Since $\kappa$ decreases in magnitude as bandgap increases [6], photovoltaic cells from wide bandgap materials can operate at higher intensity (and hence higher temperatures) than cells from narrow-bandgap materials [1,2]. For an array designed to operate at high temperature, it is also a requirement that the solar cell not physically degrade at high temperatures, for example, from degradation of the semiconductor, or from ohmic contacts diffusing through the junction. Ohmic contacts that are stable at high operation temperatures have been demonstrated for $\mathrm{GaAs}$ solar cells $[7,8]$, but work remains to be done for other semiconductor technologies. 
Likewise, other components of the system, such as the coverglass adhesive and the interconnect, must be stable at high temperature operation.

\section{APPROACHES TO SOLAR ARRAYS FOR NEAR SUN MISSIONS}

Approaches to solar arrays for near-sun missions include modifying any of the terms governing temperature or efficiency of the cell: I, the incident intensity, $\alpha$, solar absorption or $\varepsilon$, the emissivity of the cell, or $\kappa$, the temperature coefficient. Possible approaches include:

- High epsilon/low alpha coatings

- Array off-pointing (i.e., array normal points at angle to sun)

- Partially-populated array (with missing cells

replaced with mirrors)

- Spectrally-selective reflective coatings

- Solar cells designed to operate at high temperature, with low temperature coefficient $\kappa$

- Solar arrays incorporating added thermal radiators

As an example of a mission with solar panels designed to operate at high solar flux using the technique of limiting the incident flux to the array and modifying solar absorption $\alpha$, the "MESSENGER" mission to orbit Mercury [8] has designed an array with mirrored panels to reflect $7-\%$ of the incident solar energy to limit operating temperature $[9,10]$. The mirrors incorporate a highemissivity silica coating, allowing efficient thermal radiation. These panels have now successfully operated (at the first Mercury fly-by) at distance of $0.4 \mathrm{AU}$ from the sun, and have been qualified in thermal vacuum testing to be capable of operating as close as $0.25 \mathrm{AU}$ from the sun, at an intensity of 16 times the intensity at Earth orbit.

\section{SOLAR ARRAYS WITH CONSTANT POWER AT VARIABLE HELIOCENTRIC DISTANCE}

An additional problem is presented in that most missions involve a range of intensities, requiring a power system capable of operating all the way from Earth departure through a close solar pass. There are several approaches to such a power system. One straightforward approach would be to utilize several different arrays, each one optimized for a different heliocentric distance. For example, a large solar array could be used for the highest solar distances, and then discarded, or else folded away in the shadow behind spacecraft, so that a smaller solar array optimized for higher intensity could be used. Another approach would be to progressively use increasing amounts of off-pointing, so that the area exposed to the sun decreases with distance.

The problem of adapting to multiple intensity regimes is particularly difficult if the mission is required to operate both at a near-solar approach, and also at distances farther from the sun than the Earth, for example, missions using a gravity-assist at Jupiter, solar sail missions, and missions that follow a comet from distant reaches of the solar system through close approach to the sun.

One approach is to design the array to produce a constant amount of power regardless of distance from the sun. Figures 4 and 5 shows a design which use of the fact that the angular diameter of the sun increases as the spacecraft approaches the sun. A fresnel lens array focuses light onto a light pipe to transmits the light through a shield of multiple layers of foil. At high distances from the sun, the fact that the array is a concentrator array minimizes the effect of cell operation under low intensity, low temperature (LILT) condition. As the array approaches the sun, the light pipe acts as an aperture stop. As the image of the solar disk at the focal plane gets larger, the added incident light is reflected by the mirrored plate. The solar cells are mounted on a heat spreader which allows the full backside surface to serve as a thermal radiator. The portions of the collector exposed to the sunlight are refractory material (silica or high-alumina glass for the optical elements; titanium or carbon for the structural components). The solar cells, located behind the multiple layers of metal-foil insulation, are not exposed to high temperature. This array can thus be designed to put an amount of solar intensity on the cells exactly equal to that of the peak defined above.

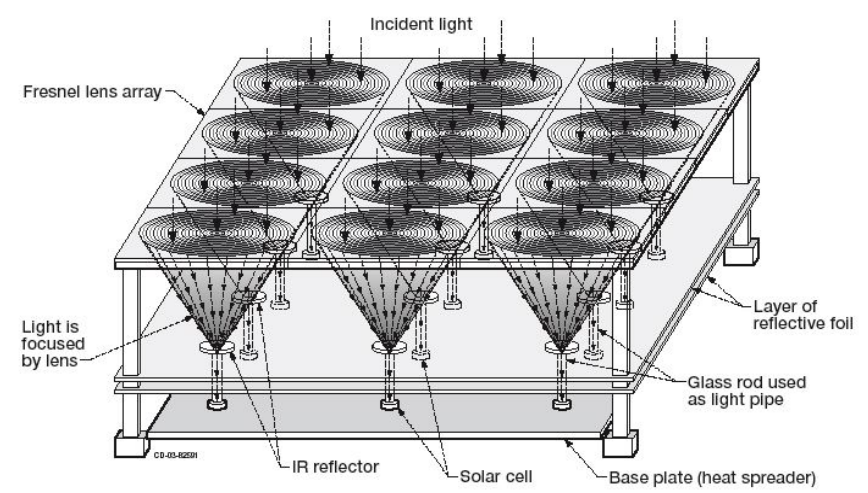

Figure 4: Concept for a concentrator solar array designed to have constant power output for variable heliocentric distances.

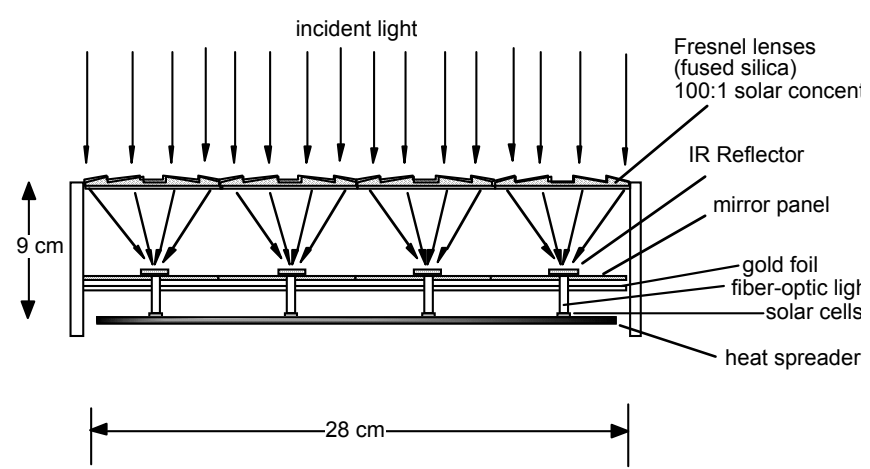

Figure 5: Cross section of concentrator solar array designed for constant power at for variable distances. 


\section{THERMAL CONVERSION FOR NEAR-SUN MISSIONS}

As a mission approaches close to the sun, the temperature of a flat-plate exposed to the sun increases. This brings out a natural question of whether it is possible to convert this heat, using a heat engine, rather than using photovoltaic arrays. Thermal power conversion is a demonstrated technology, and there exists a choice of conversion technologies, including thermoelectric, conversion, a technology with comparatively low efficiency, but high spaceflight heritage, or Stirling conversion, which has the advantage of higher efficiency but no spaceflight heritage.

Stirling converters have demonstrated 38 percent conversion efficiency [11] operating at $850^{\circ} \mathrm{C}$ hot-end, $90^{\circ} \mathrm{C}$ cold-end temperatures, which is slightly over $50 \%$ of the theoretical (Carnot) efficiency. System efficiency, accounting for other losses, is on the order of $26 \%$. These efficiency numbers are roughly comparable to the best photovoltaic technologies. The efficiency scales with temperature directly with the Carnot efficiency, proportional to the difference between the hot- and coldend temperature. Thermal conversion approaches approach would require a heat rejection radiator at the cold end of the system, which must be not exposed to the sun, either in the shadow of an absorber or the thermal shield, or else edge-on to the incident flux. The radiator must be sized to radiate the waste energy at the cold-side temperature.

Figure 6 shows the block diagram of the energy flow in such a thermal system. Since the power produced is proportional to the conversion efficiency $\eta$, and the radiator area proportional to the waste power radiated, which is proportional to $(1-\eta)$, the radiator area $A_{r}$ depends sensitively on the conversion efficiency and the radiator temperature

$$
A_{r}=[(1-\eta) /(\eta)] /\left(\varepsilon \sigma T^{4}\right) P_{\text {electric }}
$$

Emissivity $\varepsilon$ for thermal radiators is typically very close to 1 . If the radiator is two-sided, the area $A$ includes both front and rear sides.

The same equation for radiator size can be used if the thermal radiator is used to cool a solar array instead of a heat engine.

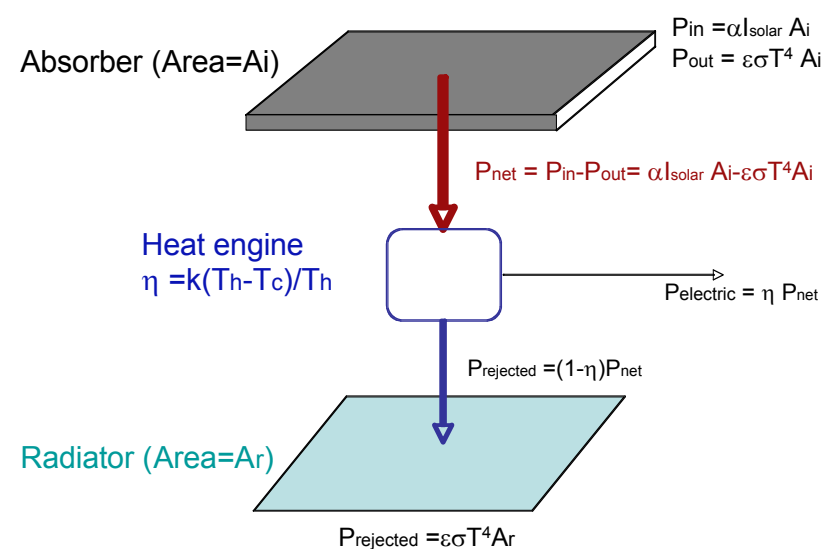

Figure 6: Thermal conversion energy flow block diagram
The thermal system chosen must operate over wide range of intensities, and hence temperatures. This can represent an engineering challenge for many missions; for example, the Solar Probe is only at close approach to the sun for a duration on the order of 10 hours.

The proposal to operate a thermal conversion system, incorporating a radiator with pumped-cooling to achieve the cold-side temperature, brings up the possibility of using a similar cooling loop to keep a solar array within operating temperature limits, thus reducing the requirement for high-temperature operation.

\section{SOLAR PROBE PLUS}

The revised mission concept for the Solar Probe mission, now renamed Solar Probe+, incorporates multiple Venus flybys to drop in to a low-perihelion orbit. The primary mission will use seven Venus flybys to bring its orbit incrementally closer to the sun, making 24 nearsun passes over a period of 6.9 years, with a final objective of a perihelion distance of roughly 9.6 solar radii, or $0.044 \mathrm{AU}$, significantly inside the orbit of Mercury. This will be about eight times closer than any spacecraft has previously approached the sun.

The new mission design incorporates several of the methods of decreasing sensitivity to multiple intensities. It uses power supply solution. The conventional and highintensity solar arrays can be seen in figure 1 .

Conventional solar array: The main array is used from Earth to slightly inside the Mercury orbit. Since the MESSENGER array is already demonstrated in flight, no technology cell or materials development is needed. The array can be offpointed from the sun to allow operation in to approximately $0.25 \mathrm{AU}$, an intensity of 16 suns. At this distance the main solar array is folded in behind the shadow shield, where it is not exposed to high temperatures, and a second power supply is used.

High-intensity solar array: The high intensity power supply is required for operation inside $0.25 \mathrm{AU}$. This power supply uses high-efficiency triple-junction solar cells designed for high solar concentrations. In order to keep the solar cells within the nominal temperature limits, a liquid cooling loop pumps coolants through thermal radiators mounted on the outside of the spacecraft. In addition, the solar array is progressively retracted behind the solar shield as it approaches the sun, in order to keep the total incident power level on the array (and hence the heat load to the radiators) approximately constant.

Details of the solar array design solution are to be presented in other papers [5].

\section{FUTURE DEVELOPMENT}

A significant driver of the size, mass, and complexity of the high-intensity power system is the size of the thermal radiator. Since the radiator area is inversely proportional to the fourth power of the radiator temperature, raising the allowable operating temperature of the solar cells has a very large effect on the radiator area. Tests are being performed to determine whether elevated operating temperatures can be tolerated by 
commercially-available solar cells without degradation.

Since light that is not absorbed by the solar array is does not contribute to the waste heat rejected by the radiator, it is advantageous to reflect light in the wavelengths not used by the cell. Most importantly, infrared light of energy less than the bandgap energy of the bottom subcell contains significant energy but makes no contribution to the output power. Blue-red rejection (BRR) filters could enhance the performance of the radiators by reflecting this unused band. The three subcells of a multijunction cell contribute roughly equally to the temperature coefficient, although the bottom cell is only a small contributor to the overall efficiency. Thus, it is likely that even more heat rejection could be accomplished if the bottom $(\mathrm{Ge})$ cell were omitted entirely from the stack, essentially returning the technological approach toward the earlier dual-junction cell technology. This would allow all the energy of wavelength longer than about $850 \mathrm{~nm}$ to be reflected away by an infraredrejection (dichroic) filter. Since the controlling parameter for the radiator sizing is the efficiency of conversion of the light absorbed by the cell, not the overall solar conversion efficiency, some reflection of the above-bandgap spectral range is acceptable, and thus the filter does not need a sharp cutoff at the band edge. This means that a thin metal film (for example, gold) or a transparent conductor could be a good choice for infrared rejection.

\section{CONCLUSIONS}

Operation of photovoltaic arrays at the high solar intensity conditions of near-sun missions presents unique challenges to solar cell technology. If the temperature of an array is allowed to vary with the thermal-radiation limit, the power produced by an array will increase as the incident flux increases up to some limit determined by the temperature coefficient and thermal properties of the solar cell, and above that output power will decrease as incident intensity increases.

A number of approaches are possible to mitigate the effects of high temperature operation.

\section{ACKNOWLEDGEMENTS}

The section of this paper about the Solar Probe + arrays incorporates information from the Solar Probe+ mission study [4], lead by the Johns Hopkins Applied Physics Laboratory. I would like to acknowledge the design work of APL in this effort, particularly James Kinnison, Martin Fraeman, Lew Roufberg, Steve Vernon, and Melissa Wirzburger.

\section{REFERENCES}

1. D. Merritt, S. Houlihan, R. Rafaelle, and G. Landis, "Wide Bandgap Space Solar Cell Development," Proc. 21st. IEEE Photovoltaic Specialist's Conference, 2005, pp. 552-555.

2. G. A. Landis, P. Jenkins, D. Scheiman, and R. Rafaelle, "Extended Temperature Solar Cell Technology Development," Proc. 2nd International Energy Conversion
Engineering Conference, paper AIAA-2004-5578, August 16-19 2004, Providence, RI.

3. G. A. Landis, R. Rafaelle, and D. Merritt, "High Temperature Solar Cell Development," Proc. 19th European Photovoltaic Science \& Engineering Conference, 2004, pp. 3659-3661.

4. J. Kinnison, et al., "The Solar Probe+ Mission: A New Concept for Close Solar Encounters", 59th International Astronautical Congress, Glasgow, Scotland, Sept. 29-Oct. 3, 2008.

5. G. A. Landis, P. C. Schmitz, J. Kinnison, M. Fraeman, L. Roufberg, S. Vernon, and M. Wirzburger, "Power System Design for the Solar Probe+ Mission," paper AIAA-2008-5712, 6th AIAA International Energy Conversion Engineering Conference, Cleveland $\mathrm{OH}$, July 28-30, 2008.

6. J. C. C. Fan, "Theoretical Temperature Dependence of Solar Cell Parameters," Solar Cells, 17, 1986, pp. 309315.

7. S. P. Tobin, et al., "Advanced Metallization for Highly Efficient Solar Cells," Proc. 19th IEEE Photovoltaic Specialists Conference, 1987, pp. 70-75.

8. M. B Spitzer, et al., "Gallium Arsenide Concentrator Solar Cells with Highly Stable Metallization," Proc. 20th IEEE Photovoltaic Specialists Conference, 1988, pp. 930933.

9. A. G. Santom, et al., "The MESSENGER mission to Mercury: Spacecraft and Mission Design," Planetary and Space Science, 49, No. 14-15, Dec. 2001, pp. 1481-1500

10. G. Dakermanji, J. Jenkins, and C. J. Ercol, "The MESSENGER Spacecraft Solar Array Design and Early Mission Performance," Proc. 2006 IEEE 4th World Conference on Photovoltaic Energy Conversion, Vol. 2, 2006, pp. 1919-1922.

11. J. Chan, J. Wood, and J. Schreiber, "Development of Advanced Stirling Radioisotope Generator for Space Exploration," Space Technology and Applications International Forum Conference, 2007, NASA/TM-2007214806. 